\title{
High-Dose Vitamin D3 Intake Is Associated with Decreased Symptoms of Multiple Sclerosis
}

\author{
${ }^{1}$ Michelle L. Steinwart and ${ }^{1,2}$ David M. Duriancik \\ ${ }^{I}$ Department of HEV, Nutrition and Dietetics Program, Central Michigan University, \\ 106 A Wightman, 1200 S. Franklin St. Mount Pleasant, MI 48859, USA \\ ${ }^{2}$ Department of Biology, University of Michigan Flint, \\ 459 Murchie Science Building, 303 E. Kearsley St. Flint, MI 48502, USA
}

Article history

Received: 29-03-2018

Revised: $17-05-2018$

Accepted: 31-08-2018

Corresponding Author: Michelle L. Steinwart Department of HEV, Nutrition and Dietetics Program, Central Michigan University, 106A

Wightman, 1200 S. Franklin St. Mount Pleasant, MI 48859,

USA

Tel: 2243307666

Email: stein5ml@cmich.edu

\begin{abstract}
Multiple sclerosis (MS) is one of the most widespread disabling autoimmune neurological conditions of adults between the ages of 20 and 40 globally. Medical researchers do not completely understand the possible causes of MS, nor have they determined the rate of progression. A limited number of clinical trials conducted in recent years have explored the link between vitamin D deficiency and MS with vitamin D supplementation as a possible element in the treatment of this disease. The primary goal of this review was to synthesize the evidence regarding the link between vitamin D3 levels and the symptoms of MS. A PubMed search was conducted using keywords Vitamin D, multiple sclerosis, MS, RRMS, prevention, treatment and cause. 1153 articles and sources were found using the key phrase "multiple sclerosis and vitamin D," but these were narrowed to 11 based on publication dates between 2013 and 2018, clinical trials were included, while reviews were excluded and the relevance of the goals to this review. Study designs included experimental clinical trials where pretest/posttest data were presented. Articles were excluded if they were not peer reviewed or only described the method and were awaiting results. Although not all studies found uniform results, the majority of the evidence suggests that high intakes of vitamin D may be associated with improved quality of life through the reduction of certain symptoms of MS. This was especially evident in patients who started the studies with a vitamin $\mathrm{D}$ deficiency. It may be too early to prescribe an increase in daily supplementation of vitamin D with the hope of reducing the development of or in the treatment of MS, but recent studies indicate that high doses of vitamin D could decrease the probability of some symptoms of the disease and possibly give favorable results in treatment. Further studies are needed before specific recommendations can be made.
\end{abstract}

Keywords: Multiple Sclerosis, Vitamin D, MS, RRMS, Prevention, Treatment, Cause

\section{Introduction}

It is estimated that 400,000 people in the United States and about 2.5 million people worldwide have been diagnosed with multiple sclerosis (MS). According to the Multiple Sclerosis Foundation, approximately 200 new cases are diagnosed each week in the United States. Adding to the urgency for prevention and cure, if a person has a parent or sibling with MS, they have a 1-3\% chance of developing the disease. MS is one of the most widespread disabling autoimmune neurological conditions of adults between the ages of 20 and 40 globally (MCS, 2014). Medical researchers do not completely understand the possible causes of MS, nor have they determined the rate of progression. Studies have led to the hypothesis that there is a genetic predisposition for MS onset, but development is increased with environmental or viral factors. There are a 
limited number of clinical trials that have been conducted in recent years that investigated the link between vitamin D deficiency as a possible cause of MS and vitamin D supplementation as a possible element in the prevention and treatment of this disease (MCS, 2014; MSF, 2018; MSAA, 2014).

Multiple Sclerosis (MS) has been defined as a chronic inflammatory demyelinating disease that affects the central nervous system (O'Connell et al., 2017). While researchers have not yet found a clear cause for developing MS, it is categorized as an autoimmune disease. In MS, the body's immune system invades the myelin coating of the nerve fibers and disrupts communication between the brain and the body. Ultimately, MS can cause the nerves to degrade or become irreparably damaged (MCS, 2014).

The amount of nerve damage and the specific nerves that are affected dictate which symptoms are manifested. Severity may range from the loss of ability to walk independently to extended periods of remission.1 According to Lassmann and Kulselnigg (2014), MS can even affect the bones of the body and can lead to osteoporosis. Several studies have shown that people with Multiple Sclerosis (MS) have an increased risk of developing osteoporosis (Huang et al., 2015). Lack of physical activity is likely the main cause of accelerated bone loss in MS, but also duration of the disease and long term steroid use are linked with low Bone Mineral Density (BMD) (Steffensen et al., 2010). However, low BMD is also prevalent in ambulatory individuals with MS even shortly after clinical diagnosis (Moen et al., 2011), suggesting that common etiological components such as low vitamin D may be at work in both MS and osteoporosis. The combination of osteoporosis and an increased risk of falling might add to the burden of MS through elevated risk of fractures. Large population based studies have indicated that individuals with MS have a significant increase of fractures compared to the general population (Bazelier et al., 2011). Data from The National Hospital Discharge Registry showed that the risk of fractures in individuals with MS was three to six times higher than what is seen in the general population (Bazelier et al., 2012).

Other observable symptoms may include limb weakness that often happens on one side of the body, partial or complete reduction of vision, often in one eye at a time, painful eye movement, long term double vision, extremity pain or tingling, electric shock feelings that happen when the neck is moved in certain positions, tremor, lack of coordination or unsteady gait, slurred speech, fatigue, dizziness and problems with bowel and bladder function (MCS, 2014).

These symptoms can appear in intermittent phases throughout a person's life. This is called relapsing remitting MS (RRMS) and can eventually lead to progressive MS. The majority of people with MS experience a relapsing remitting disease course, where they have different symptoms that manifest over a period of time and then partial or complete improvement. Relapses can last for months or possibly years (MCS, 2014).

While it is not clear why MS occurs in some people and not others, a combination of genetic and environmental factors could be responsible (MCS, 2014; Lassmann and Kulselnigg, 2014). There are some factors that might affect this progression, such as skin phenotype and geographic location, which dictates sunlight exposure levels and possible deficiencies of vitamin D. Risk factors that have been considered include geographic location/climate, age, gender, race, family history and smoking (MCS, 2014).

Adding to the difficulty and strain of tolerating a debilitating disease such as MS is the fact that it is an expensive disease to treat. Obvious and more hidden health care costs range from $\$ 8,528$ to $\$ 54,244$ per patient per year in the United States. MS ranks second only to congestive heart failure in terms of costliness compared with other chronic conditions (Adelman et al., 2013). These high health care costs add to the urgency to find a less expensive treatment path. Currently, there is no recognized cure for MS. However, some available treatments can increase speed of recovery from attacks, alter the path of the disease and manage the various symptoms. Some researchers hypothesize that vitamin D deficiency could be central to this disease and the inexpensive supplementation of vitamin D could lead to prevention and potentially a cure (Schlogol and Holick, 2014). This vitamin has been drawing the attention of researchers for several decades, but with the growing number of cases reported every year, the importance for continued research in this area has increased.

According to Mayo Clinic, vitamin D is found in a variety of foods, including eggs, fish, fortified milk and cod liver oil. Sunlight also contributes to vitamin D status and approximately $10 \mathrm{~min}$ of sun exposure per day is adequate to help avoid a deficiency at certain latitudes and times of the year (MCS, 2017). The primary role of vitamin $\mathrm{D}$ is to preserve normal levels of phosphorus and calcium. Vitamin D assists the body in calcium absorption, which builds and preserves strong bones. Vitamin D can be used with calcium to improve overall bone health and reduce fractures. This vitamin could also guard against osteoporosis, cancer, high blood pressure and other diseases (MCS, 2017). An estimated 1 billion people throughout the world have deficient levels of vitamin D (Holick, 2007).

Although more research is required prior to recommending an increase in daily supplementation of vitamin $\mathrm{D}$ with the intention of reducing the development of or providing treatment for MS, recent studies demonstrate that extremely low levels of vitamin $\mathrm{D}$ increase the likelihood of a positive diagnosis of the disease and high doses improve treatment results. 


\section{Method}

A PubMed search was conducted using keywords Vitamin D, multiple sclerosis, MS, RRMS, prevention, treatment and cause. 1153 articles and sources were found using the key phrase "multiple sclerosis and Vitamin D," but results were narrowed to 11 based on publication dates between 2013 and 2018 and the relevance of goals to this review. Study designs included experimental clinical trials where pretest posttest data were presented. Articles were excluded if they were not peer reviewed or only described method and were awaiting results.

\section{Results}

Initially, 1153 articles were identified. Based on the titles and abstracts, 35 articles were retrieved for full text review. Finally, a total of 11 original articles qualified to be reviewed. A summary of studies on the association between vitamin $\mathrm{D}$ supplementation and symptoms of MS is presented in Table 1. The studies included in this review presented with varied results.

Ashtari et al. (2016) argued that low serum levels of vitamin $\mathrm{D}$ are linked to a more severe disease path and also decreased quality of life in Relapsing Remitting Multiple Sclerosis (RRMS) and that intake of high dose vitamin D improves perceived quality of life in RRMS patients. The authors investigated the question of whether high dose vitamin D intake has any effect on the quality of life of RRMS patients. They gave one group of the 94 RRMS patients 50,000 UI vitamin D3 every five days for three months, while the other group received a placebo. At the end of the study, the group that received vitamin $\mathrm{D}$ had a significant difference in mental health as their mental quality of life scores improved significantly (Ashtari et al., 2016).

Table 1: Studies focused on RRMS symptoms and vitamin D3

\begin{tabular}{llll}
\hline Study, year, study design & Sample used & Outcome measure & Results \\
\hline Holmoy et al (2017) & 68 persons with RRMS; 20,000 IU & Measured serum D3 levels and & Neither PINP nor CTX1 predicted \\
$\begin{array}{l}\text { Randomized controlled } \\
\text { trial }\end{array}$ & $\begin{array}{l}\text { D3 given weekly to half of group, } \\
\text { placebo to other half over 96 weeks }\end{array}$ & $\begin{array}{l}\text { parathyroid hormone at week 48 } \\
\text { and week 96. Bone formation } \\
\text { measured by procollegen type }\end{array}$ & $\begin{array}{l}\text { bone loss from baseline to week 96. } \\
\text { bone resorption. }\end{array}$
\end{tabular}
asured by procollegen type

$1 \mathrm{~N}$ propeptide (PINP). Bone resorption measured by $\mathrm{C}$-terminal cross-linking telopeptide of type 1 collagen (CTX1).

O'Connell et al (2017) 41 healthy adult controls and 49 single center, investigator-led, with clinically isolated syndrome exploratory, double-blind,

randomized, placebo controlled

Laursen et al (2016)

prospective cohort study

Muris et al (2016b) exposed/ randomized controlled trial

Muris et al (2016a) retrospective follow-up study
170 RRMS adults: natalizumabtreated with no control group

30 Dutch RRMS adults being treated with IFN-Beta-1a. Received high dose D3 and 23 controls received placebo over 48 weeks. About $554 \mathrm{MS}$ patients with serum baseline 25(OH)D levels and Expanded Disability Status Scaleminimum follow-up of 3 years
Frequency of pro-inflammatory CD4+ T cells and level of disease freedom with 5000 IU or 10,000 IU of D3.

Measured relapse rate over time; clinical effects associated with D3 levels Changes in immune regulatory parameters:

Effect of baseline D3 levels on relapse rate. Repeated measures linear regression analysis performed to assess effect on disability and disability progression.

Quality of Life (MSQOL-54) was assessed at baseline and end of

Double-blind randomized clinical trial

Toghianifar et al (2015) Double-blind randomized clinical trial

Ashtari et al (2015) double-blind randomized clinical trial

Fitzgerald et al (2015) prospective cohort study
94 patients with RRMS receiving interferon- beta therapy: Randomized into 2 groups. One group received 50,000 IU D3 every 5 days for three months. The other received placebo.

94 RRMS subjects on IFN-Beta therapy in two groups: 50,000 IU

D3 every 5 days for 12 weeks and placebo group

94 patients with RRMS receiving interferon therapy; randomized into treatment and placebo groups Treatment group received 50,000 IU D3 every 5 days for 3 months

1482 MS patients. received dose

of interferon- $1 \mathrm{~b}$ ( 250 or 500 units) with at least 2 measurements of $25(\mathrm{OH}) \mathrm{D}$ obtained 6 months apart. study.

Serum levels of IL-17 measured at the beginning of study and after 12 weeks.

IL-10 measured at baseline and after 3 months

Monitored for 2 years. Clinic visits every 3 months. MRI performed at baseline and then annually to measure MS disease progression
No difference noted in proinflammatory CD4+ T cells. A higher level of disease freedom was seen but not at significant level.

D3 supplementation significantly associated with decreased relapse rate

After 48 weeks, there was not an increase in lymphocytes with a regulatory phenotype.

Baseline 25(OH)D status was associated with subsequent relapse risk, but only in younger MS patients. Baseline 25(OH)D status not significantly associated with either disability or disability progression, irrespective of MS phenotype.

After three months, D3 group had significant difference in mental health composite compared to placebo group.

IL-17 levels showed significant change in RRMS patients after receiving high dose D3 for 12 weeks

IL-10 levels increased significantly after receiving high-dose D3 for 3 months

Average 25(OH)D levels were significantly inversely correlated with cumulative number of new active lesions. No significant 
Table 1: Continue

Gargari et al (2015) clinical trial non-controlled
32 RRMS patients received 50,000 IU D3 every week for 2 months; no control group
Rosjo et al (2015) Two-year double-blind placebocontrolled randomized clinical trial

\section{2 adult RRMS patients}

randomized into 2 groups: 20,000

IU D3 weekly and placebo groups over 96 weeks

\section{(lesions). Expanded Disability Status Scale was also used to measure progression. \\ Blood work to compare gene expression before and after supplementation of D3. IL-6 and IL-17A expression levels.}

\section{Examined 11 serum markers of} inflammation, bone health and clinical disease parameters.

\author{
associates found between $25(\mathrm{OH}) \mathrm{D}$ \\ levels and change in brain volume, \\ relapse rate, or EDSS scores. \\ Significant up-regulation of IL-6 and \\ IL-17 gene expression shown with \\ D3 treatment. Some gender specific \\ correlations found between the gene \\ expression with D3 levels in female \\ RRMS patients. \\ No difference in decline in bone \\ mass, clinical disease activity/ \\ progression between groups. BUT \\ mean serum 25(OH)D levels more \\ than doubled for those receiving the \\ supplements.
}

In another study, Ashtari and colleagues examined the effects of high dose vitamin D on interleukin 10 (IL-10) levels in MS with a sample of 94 RRMS patients who were randomly grouped into a treatment and placebo group. The two groups received conventional MS treatments, but the treatment group also received 50,000 IU of vitamin D every five days for three months. At the end of the study, the researchers found that IL-10 levels increased significantly after taking the high-dose vitamin D3 for three months. They concluded that high doses of vitamin $\mathrm{D}$ could be valuable in promoting an anti-inflammatory state in RRMS patients (Ashtari et al., 2015).

Fitzgerald et al. (2015) studied the relationship between 25-hydroxyvitamin D (25[OH] D) levels and disease course in patients with RRMS treated with interferon beta-1b. The two-year research study included 1482 patients who were participants in the Betaferon Efficacy Yielding Outcomes of a Dose (BEYOND). At the beginning of the study, an MRI was completed and then repeated annually. The patients visited the clinic every three months and received $250 \mu \mathrm{g}$ or $500 \mu \mathrm{g}$ of interferon-1b. They also had at least 2 measurements of $25(\mathrm{OH}) \mathrm{D}$ obtained 6 months apart. The investigators examined the association between the $25(\mathrm{OH})$ D levels and MS outcomes in both longitudinal and cross sectional analyses. They found no major association in a cross sectional analysis between baseline 25(OH)D levels and brain volume, total number of relapses, or changes in disability scale scores.

Additionally, the patients with higher serum $25(\mathrm{OH}) \mathrm{D}$ levels during the first 12 months of the study developed fewer new active lesions observed on MRI during a median followup time of 2 years. This inverse longitudinal association between 25(OH) D levels and MS activity was consistent with and independent of HLA-DRB1 * 15 or vitamin Dbinding protein status and was present across various subgroups of patients with different baseline characteristics. The authors concluded that vitamin D levels above the currently recommended level could be beneficial to MS patients.
Holmoy et al. (2017) pursued the questions of whether low vitamin D levels were related to an increased future risk of developing MS and if vitamin D supplementation should be recommended both to prevent MS progression and to maintain bone health. This hypothesis was fueled by the findings from past studies which indicate MS patients have a higher risk of developing osteoporosis. Additionally, people with MS have also been found to have an increased risk of fractures as compared to the overall population. For this study, the team identified 71 eligible subjects but eliminated some due to a history of diseases affecting the bones, pregnancy, or breastfeeding for the last six months. The controlled study was completed with 68 randomized Norwegian subjects aged 18 to 50 years. The participants received either 20,000 IU D3 once per week orally or a placebo. All participants also received $500 \mathrm{mg}$ of calcium daily. Bone Mass Density (BMD) measurements and serum samples for measurement of bone markers were obtained at baseline, at 48 and at 96 weeks. Using linear regression, the team analyzed the associations of bone markers and BMD at baseline and whether concentrations of PINP and CTX1 at the beginning of the study predicted BMD variations at the end of the study.

The researchers noted an increase in the serum concentration of $25(\mathrm{OH}) \mathrm{D}$ to $123.2 \pm 34.2 \mathrm{nmol} / \mathrm{L}$ at week 96 in the vitamin $\mathrm{D}$ group and to $61.8 \pm 25.2 \mathrm{nmol} / \mathrm{L}$ in the placebo group. The calcium level remained unchanged. They found that MS patients who took vitamin D supplements did not improve the BMD. The investigators found no effect on bone formation as measured by procollagen type I N propeptide or on bone resorption. They concluded that the supplementation of vitamin D was not helpful to people who have MS. Additionally, vitamin D was found insufficient in the prevention of bone loss in MS patients who were not deficient in the vitamin at baseline (Holmoy et al., 2017).

Based on the thought that the risk of developing MS and disease activity is associated with vitamin D status, Muris et al. (2016a) studied 554 MS patients with a 
serum baseline 25(OH)D level and Expanded Disability Status Scale (EDSS) with a minimum followup of three years. Participants had baseline serum 25(OH)D levels measured as well as an EDSS within six months. Data relating to MS was collected on all clinical visits. Serum $25(\mathrm{OH}) \mathrm{D}$ levels were measured through routine clinical analysis. The team performed logistic regressions to assess $25(\mathrm{OH}) \mathrm{D}$ status on relapse rate. Repeated measures linear regression analyses were implemented to determine the effect on disability and disability progression. They examined the primary outcome variables which were the relation of $25(\mathrm{OH}) \mathrm{D}$ status with relapses, the relation with EDSS and the relation with EDSS progression.

The researchers found that within the physiological range, the $25(\mathrm{OH}) \mathrm{D}$ status is only significantly related to the occurrence of relapses in the younger MS patients. It was, however, not correlated with the disability or its progression over a three year follow up. They did not find any evidence to support whether vitamin D affects disability progression in MS patients. The researchers recommend further long term follow up of supplementation studies to determine whether high-dose supplementation to supraphysiological $25(\mathrm{OH}) \mathrm{D}$ levels prevents disability progression in MS. They advised that young MS patients should be given vitamin D3 supplements as a preventative option.

Muris et al. (2016b) investigated the effects of highdose vitamin supplementation on a group of 30 Dutch RRMS patients who had been treated with IFN $\beta-1$ while 23 patients got a placebo. They found that the number of cells in the immune regulatory compartment remained unaltered when patients were given a high dose of vitamin D3. The researchers concluded that supplementing vitamin D3 did not equate to an increase in lymphocytes with a regulatory phenotype. However, they did find that vitamin $\mathrm{D}$ contributed to the preservation of immune homeostasis through prevention of more disturbance in the T cell compartment in the onset of MS.

Gargari et al. (2015) investigated the effects of vitamin D on the expression level of IL-6 and IL-17A in Peripheral Blood Mononuclear Cells (PBMCs) of patients with MS. Additionally, they carried out a correlation between the gene expression and some clinical features such as serum level vitamin D and the Expanded Disability Status Scale (EDSS). Significant up regulation of IL-6 and IL-17A gene expression was shown under vitamin $\mathrm{D}$ treatment. The team also detected some gender-specific correlations between the gene expressions with vitamin $\mathrm{D}$ levels in female patients.

The researchers found that IL-6 and IL-17A gene expression up-regulate under vitamin D treatment but no correlation was observed between EDSS scores with IL6 and IL-17A expression. It was also evident that some MS patients did not respond to vitamin D supplementation and that other medications being taken can explain why this occurs. No placebo-control group was used in this study and the sample size was very small. The authors admit that a longer period of supplementation with different doses could help identify the exact roles of vitamin D in MS.

O'Connell et al. (2017) set out to investigate the immunologic effects of vitamin D in both healthy individuals and MS patients with Clinically Isolated Syndrome (CIS). Their primary goal was to show the positive immunologic effects of two doses of vitamin D compared with a placebo over 24 weeks in both groups. The study was conducted using 39 healthy participants and 32 CIS patients who were randomized into either a vitamin D 5,000 IU daily, vitamin D 10,000 IU daily or a placebo. The participants had no history of renal impairment, vitamin D intolerance, hypercalcemia, or parathyroid dysfunction. The CIS patients must not have had an occurrence of an exacerbation less than six weeks before study entry, previous treatment with $\beta$-interferons or glatiramer acetate in the three months before study entry, previous treatment with steroids in the four weeks before study entry, or any previous treatment with mitoxantrone among others to qualify. Female participants were asked not to get pregnant or use effective contraception in the course of the study and three months following the study.

The main outcome goal upon completion of the study was immunologic measures of the frequency of CD4 Tcell subsets and cytokine responses in peripheral blood mononuclear cells, assessed at baseline and after 16 and 24 weeks of treatment. The total runtime of the study was 24 weeks, during which the subjects provided a medical history, list of medications and had a physical and neurological examination. The patients were assessed for relapse evidence, defined as the appearance of new neurological abnormality or reappearance of a previously seen neurological abnormality. Any defect must have been present for $24 \mathrm{~h}$, be based on objective clinical evidence and must have happened in the absence of any infection or fever. The authors analyzed serum vitamin D levels and parathormone levels at baseline and also each month for a total of 5 months by liquid chromatography mass spectrometry. A baseline brain MRI was conducted at the beginning and at the end of 5 months. The testing was done without the two radiologists' knowledge of the treatment group to which the participant belonged. The authors concluded that supplementation of Vitamin D3 up to 10,000 IU daily was safe and tolerable in both healthy and CIS patients. High doses of vitamin D3 did not reduce proinflammatory IL-17+CD4+ $\mathrm{T}$ cells. They further concluded that the possible role of D3 as an immunomodulator would require additional investigation in larger randomized controlled clinical trials over a longer period of time. 
Rosjo et al. (2015) studied the possible antiinflammatory effects of vitamin D in RRMS patients by examining the impact of high-dose oral vitamin D3 on eleven markers of systemic inflammation. Using 68 subjects, they measured serum inflammation markers and 25-hydroxyvitamin at baseline and again at week 96 . They found no significant difference between the vitamin D group and the placebo group for any of the inflammation markers. They concluded that a high-dose vitamin D3 supplementation significantly increased serum $25(\mathrm{OH})$ D levels without affecting markers of systemic inflammation. The researchers, however, did not dismiss the idea that vitamin D3 supplementation could cause short-term effects on the markers that were measured or affect other systemic markers that were not included in this study such as IL-6 and IL-10.

Toghianifar et al. (2015) analyzed the effect of highdose oral vitamin D intake on IL-17 levels in MS patients in a double-blind, randomized clinical trial. Past studies have shown that vitamin $\mathrm{D}$ reduces the number of IL-17 secreting cells. In this study, the team wanted to compare serum levels of IL-17 in MS patients taking high-dose vitamin $\mathrm{D}$ with a placebo group. The participants had to be diagnosed with RRMS, aged between 18 and 55 years, free of relapse 30 days before the study commenced, had to have an EDSS score of less than 4 and negative $\beta-\mathrm{HCG}$ test for women in childbearing age. Using 94 patients with RRMS divided randomly into two groups, they gave one group 50,000 IU vitamin D3 every five days for 12 weeks while the other group received a placebo. Both groups also received Interferon- $\beta$ (IFN- $\beta$ ) treatment. Serum levels of IL-17 were measured at the beginning of the study and after 12 weeks. The treatment group received 50,000 IU of vitamin D every five days with regular visits after 4,8 and 12 weeks. Data was summarized using median and inter-quartile range. Through the multiple linear regression analysis, they found that $25(\mathrm{OH}) \mathrm{D}$ levels increased significantly for the treatment group compared to the placebo group. They concluded that the consumption of vitamin D3 was positively and significantly associated with the logarithm of IL-17 measures and concluded that IL-17 levels presented a significant change in patients after receiving high-dose vitamin D3 for 12 weeks. They also recommended extensive studies to explain the role of vitamin D on IL17 levels and whether it has any effect on disease activity and course while considering probable confounding parameters such as dose and duration of vitamin D supplementation.

Laursen et al. (2016) investigated the use of vitamin D supplements in treating patients with MS in temperate climates. Their study is based on the qualities of vitamin $\mathrm{D}$ as a protective agent against diseases in children who are developing their immune system. Their research focused on the immunomodulatory role of vitamin D and how it relates to lymphocytes to boost the neuro protective mechanism of the body. By administering vitamin D supplements to 134 natalizumab treated RRMS patients from an out patient clinic during two consecutive winter seasons, the authors found a significant reduction in relapses and an improved quality of life only for those who had vitamin D3 deficiency at baseline. Those participants started showing fewer signs and symptoms by continued intake of the supplements. Improvement, however, was not observed for patients with normal baseline D3 levels. The researchers concluded that increases in D3 levels in the groups who were not D3 deficient at the start of the study were too small to affect the clinical outcome. No placebo control group was used in this study and the authors admit that the natural path of MS may influence the results over time.

\section{Discussion}

This review assessed 11 original studies on the association between vitamin D and the symptoms of Multiple Sclerosis (MS) in adult patients and highlighted that although not all studies showed consistent results, the majority found an inverse association between high doses of vitamin D and the symptoms of MS.

Five of the studies reviewed reported significant results when high dosages of vitamin D3 were administered (Ashtari et al., 2015; 2016; Gargari et al., 2015; Toghianifar et al., 2015; Laursen et al., 2016). These improvements were seen when dosage was set at $50,000 \mathrm{IU}$ and manifested in a decrease of relapse rate; up-regulation of IL-6, IL-10, IL-17; and in increased quality of life. All of these studies had a duration of three months but differed in sample size and study design. Three of the studies had a sample size of 94 patients with RRMS (Ashtari et al., 2015; 2016; Toghianifar et al., 2015), while one study examined 170 patients (O'Connell et al., 2017) and the other only 32.11 The studies conducted by Laursen et al. (2016) and Gargari et al. (2015) did not use a control group. The other three studies were double blind, randomized control trials. Gargari and colleagues noted that some gender specific correlations were found between gene expression with vitamin D3 levels in female RRMS patients. The other studies made no note of gender specificity. Also, while Toghianifar et al. (2015) noted that high dose D3 was well tolerated and IL-17 levels showed significant change, they questioned the effect it had on disease activity and progression.

While the above studies reported encouraging results, four studies in this review showed results that were not as positive (O'Connell et al., 2017; Holmoy et al., 2017; Muris et al., 2016b; Rosjo et al., 20115). Holmoy et al. (2017) and Rosjo et al. (2015) examined bone health in RRMS patients who received 20,000 IU D3 weekly over 
96 weeks. Both groups found that there was no difference in bone mass decline, bone formation, or on bone resorption. Rosjo's group, however, did see the serum 25(OH)D levels more than doubled for the group receiving the supplements. O'Connell et al. (2017) noted no difference in pro-inflammatory CD4+ T cells and while they observed a higher level of disease freedom, it was not at a significant level. This group admitted that further study is needed in this area. One study looked at changes in immune regulatory parameters and after 48 weeks, there was no increase in lymphocytes with a regulatory phenotype (Muris et al., 2016b). However, the group believes the results support the hypothesis that D3 contributes to maintenance of immune homeostasis by preventing further disturbance of the $\mathrm{T}$ cell compartment early in the disease course of MS.

Two studies in this review showed mixed results. Fitzgerald et al. (2015) studied a large sample of MS patients over a two year period using Magnetic Resonance Imaging and a disability scale at the measurement tool. These researchers found that average serum D3 levels were significantly inversely related with the total number of new and active brain lesions, but found no significant correlation between serum D3 levels and a change in brain volume, relapse rate, or scores on a disability scale. Muris and colleagues 19 found that the baseline D3 levels of the patients in their study were associated with subsequent relapse risk, but only in the younger patients in their study. They also did not find a significant correlation with disability progression.

It should be noted that the studies included in this review were heterogeneous in nature, with diverse study populations, sample size and study length. The main strength of this review is the extensive, systematic literature search that involved clear inclusion criteria.

\section{Conclusion}

It may be too early to prescribe an increase in daily supplementation of vitamin D with the hope of stopping the development or in the treatment of MS. Recent studies indicate that high doses of vitamin D could decrease the probability of some symptoms of the disease and possibly give favorable results in treatment. Rigorous, pragmatic, randomized, controlled clinical trials are needed before recommendations can be made specifically related to the intake of D3 supplements in patients at risk for or diagnosed with MS.

\section{Author's Contributions}

All authors equally contributed in this work.

\section{Ethics}

This article is original and contains unpublished material. The corresponding author confirms that all of the other authors have read and approved the manuscript and there are no ethical issues involved.

\section{References}

Adelman, G., S.G. Rane and K.F. Villa, 2013. The cost burden of multiple sclerosis in the United States: A systematic review of the literature. J. Med. Econ., 16: 639-647. DOI: 10.3111/13696998.2013.778268

Ashtari, F., N. Toghianifar, S.H. Zarkesh-Esfahani and M. Mansourian, 2015. Short-term effect of highdose vitamin D on the level of interleukin 10 in patients with multiple sclerosis: A randomized, double blind, placebo controlled clinical trial. Neuroimmunomodulation, 22: 400-404. DOI: $10.1159 / 000439278$

Ashtari, F., N. Toghianifar, S.H. Zarkesh-Esfahani and M. Mansourian, 2016. High dose vitamin D intake and quality of life in relapsing remitting multiple sclerosis: A randomized, double blind, placebo controlled clinical trial. Neurol. Res., 38: 888-892. DOI: 10.1080/01616412.2016.1227913

Bazelier, M.T., F. de Vries, J. Bentzen, P. Vestergaard and H.G. Leufkens et al., 2012. Incidence of fractures in patients with multiple sclerosis: The Danish national health registers. Mult. Scler., 18: 622-627. DOI: $10.1177 / 1352458511426739$

Bazelier, M.T., T.P. van Staa, B.M. Uitdehaag, C. Cooper and H.G. Leufkens et al., 2011. The risk of fracture in patients with multiple sclerosis: The UK general practice research database. J. Bone Miner Res., 26: 2271-2279. DOI: 10.1002/jbmr.418

Fitzgerald, K.C., K.L. Munger, K. Kochert, B.G. Amason and G. Comi et al., 2015. Association of vitamin D levels with multiple sclerosis activity and progression in patients receiving interferon Beta- $1 \mathrm{~b}$. JAMA Neurol., 72: 1258-1465. DOI: $10.1001 /$ jamaneurol.2015.2742

Gargari, B.N., M. Behmanesh, Z.S. Farsani, M.P. Kakhki and A.R. Azimi, 2015. Vitamin D supplementation up-regulates IL-6 and IL-17A gene expression in multiple sclerosis patients. Int. Immunopharmacol., 28: 414-419. DOI: $10.1016 /$ j.intimp.2015.06.033

Holick, M.F., 2007. Vitamin D deficiency. N Engl. J. Med., 357: 266-281. DOI: 10.1056/NEJMra070553

Holmoy, T., J.C. Lindstrom, E.F. Eriksen, L.H. Steffensen and M.T. Dampman, 2017. High dose vitamin D supplementation does not affect biochemical bone markers in multiple sclerosis a randomized controlled trial. BMC Neurol., 17: 67-67. DOI: $10.1186 / \mathrm{s} 12883-017-0851-0$

Huang, Z., Y. Qi, S. Du, G. Chen and W. Yan, 2015. Bone mineral density levels in adults with multiple sclerosis: A meta analysis. Int. J. Neurosci., 125: 904-912. DOI: 10.3109/00207454.2014.988332 
Lassmann, H. and A. Kulselnigg, 2014. Pathology of multiple sclerosis and related inflammatory demyelinating diseases. Handb. Clin. Neurol., 122: 15-58. DOI: 10.1016/B978-0-444-52001-2.00002-9

Laursen, J.H., H.B. Sondergaard, P.S. Sorensen, F. Sellberg and A.B. Oturai, 2016. Vitamin D supplementation reduces relapse rate in relapsing remitting multiple sclerosis patients treated with natalizumab. Mult. Scler. Relat. Disord., 10: 169-173. DOI: 10.1016/j.msard.2016.10.005

MCS, 2014. Mayo Clinic Staff. Risk factors for MS.

MCS, 2017. Mayo Clinic Staff. Vitamin D.

Moen, S.M., E.G. Celius, L. Sandvik, L. Nordsletten and E.F. Eriksen et al., 2011. Low bone mass in newly diagnosed multiple sclerosis and clinically isolated syndrome. Neurology, 77: 151-157.

DOI: $10.1212 /$ WNL.0b013e3182242d34

MSAA, 2014. Frequently asked questions about multiple sclerosis. Multiple Sclerosis Association of America.

MSF, 2018. Fact about MS (n.d.) Multiple Sclerosis Foundation.

Muris, A.H., J. Smolders, L. Rolf, L.J. Klinkenberg and N. van der Linden et al., 2016a. Vitamin D status does not affect disability progression of patients with multiple sclerosis over three year follow up. PLoS One, 11: e1056122-e1056122. DOI: $10.1371 /$ journal.pone.0156122

Muris, A.H., J. Smolders, M. Thewissen, R. Hupperts and J. Damoiseaux, 2016b. Immune regulatory effects of high dose vitamin D3 supplementation in a randomized controlled trial in relapsing remitting multiple sclerosis patients receiving IFN Beta; the SOLARIUM study. J. Neuroimmunol., 300: 47-56. DOI: 10.1016/j.neuroim2016.09.018
O’Connell, K., J. Sulaimani, S.A. Basdeo, K. Kinsella and S. Jordan et al. 2017. Effects of Vitamin $\mathrm{D}_{3}$ in clinically isolated syndrome and healthy control participants: A double blind randomized controlled trial. Mult. Scler. J. Exp. Transl. Clin. DOI: $10.1177 / 2055217317727296$

Rosjo, E., L.H. Steffensen, L. Jorgensen, J.C. Lindstrom and J. Saltyte Bente et al., 2015. Vitamin D supplementation and systemic inflammation in relapsing remitting multiple sclerosis. J. Neurol., 262: 2713-2721. DOI: 10.1007/s00415-015-7902-5

Schlogol, M. and M.F. Holick, 2014. Vitamin D and neurocognitive function. Clin. Interv. Ag., 2014: 559-568. DOI: $10.2147 /$ cia.s51785

Steffensen, L.H., S.I. Mellgren and M.T. Kampman, 2010. Predictors and prevalence of low bone mineral density in fully ambulatory persons with multiple sclerosis. J. Neurol., 257: 410-418. DOI: $10.1007 / \mathrm{s} 00415-009-5337-6$

Toghianifar, N., F. Ashtari, S.H. Zarkesh-Esfahani and M. Mansourian, 2015. Effect of high dose vitamin D intake on interleukin-17 levels in multiple sclerosis: A randomized, double-blind, placebo-controlled clinical trial. J. Neuroimmunol., 285: 125-128.

DOI: 10.1016/j.jneuroim.2015.05.022 